\title{
A Brief Report about Managing the Spread of COVID-19 with Limited Resources in Bandar Abbas City, South of Iran
}

\author{
Hashem Jarineshin, Fereydoon Fekrat, Behnaz Dalfardi, Fatemeh Khosravi*
}

\begin{abstract}
Since the end of 2019, the world has experienced the biggest catastrophic event of the century because of the manifestation of novel coronavirus disease (COVID-19). COVID-19 caused by severe acute respiratory syndrome coronavirus 2 (SARS-CoV-2), which could lead to severe pneumonia. All countries need to prepare their resources and infrastructures as well as healthcare systems to overcome this disaster or any next pandemic, which may occur in the future again. At the time of this writing, my country is suffering from second wave of this pandemic. In Iran, the prevalence of COVID-19 with a high rate of mortality is still at an alarming rate which brings severe concern and a massive burden for health care systems. As of September 15, 2020, the number of infected people with COVID-19 in Iran was 407,353 with 23,453 deaths. In this short report, we want to share our response planning with emphasis on the measures being applied in our hospital particularly our anesthesiology department, to decrease the spread of COVID-19 in the operating rooms and intensive care units (ICU) of this significant referral centre (450-bed) of Hormozgan university located in Bandar Abbas, Hormozgan province south of Iran.
\end{abstract}

Key words: COVID-19, Coronavirus disease, Pandemic, Management, Strategies.

\section{REPORT}

A novel human coronavirus 2019 (COVID-19) is a severe, complex infectious disease which has become a significant source of death all over the world. In late December 2019, the outbreak of COVID-19 was first started from Wuhan, a city in China and after that has spread rapidly all over the world, mainly through human-to-human transmission. ${ }^{1}$ The origin of this virus was first discovered in a seafood market in Wuhan and many chines researchers suggested that bats are responsible for this unknown transmission. The mechanisms underlying the detrimental effects of COVID-19 were not fully elucidated and due to the rapid progression of this mysterious virus, the World Health Organization (WHO) announced COVID-19 is a global emergency and a pandemic situation on March $112020 .^{2}$ In Iran, the first cases of COVID19 were reported on February 19, 2020. Meanwhile, according to the report of the Ministry of Health and Medical Education on September 15,2020, the number of confirmed cases in Iran reached 407,353 and with 23,453 confirmed deaths as a consequence of COVID-19. ${ }^{3}$ Our academic hospital (Payambar Azam medical centre) is a general 450-bed university hospital in Bandar Abbas which is a famous port city for seaborne trade and transportation located on the southern coast of Iran, on the Persian Gulf. In addition to the seaborne economical activities, our city has a fabulous social and tourist attraction. The initiation of the outbreak of COVID-19 coincided with New Year holiday; as a result, a large number of domestic tourists visited our city increasing human interaction and crowding. Because of this, we anticipated a sudden, unexpected increase in the number of new cases and swift action was needed. Our hospital was chosen as a referral centre for COVID19 cases and an internal multidisciplinary protocol was developed in our centre and the capacity of the hospital was amplified first by discharging many patients from various wards to create bed capacity. In our centre, we considered some particular strategies to manage this infection. Since COVID-19 is still considered a disaster circumstance for all countries, sharing different experiences is an excellent strategy to understand better conditions and prepare all infrastructures to deal with anticipated surge which may occur again in the future. The followings are some of the examples of potential management and preparation against COVID-19 outbreak.

\section{Preparing the emergency department and rapid assessment units}

The primary strategy was reducing the risk of contamination for other parts of the hospital. Thus, for this purpose, we set up a tent (32-bed) in the area which was away from hospital centre to triage the cases (mild, moderate and severe COVID-19 cases from non-COVID-19). This early containment was our priorities to break the chain of viral infection. On 
arrival to this emergency ward, first, all patients were given a surgical mask (because many of these patients could be contagious). Then they were examined by an infectious disease specialist, applying a through surveillance. The patients who met the diagnostic criteria (fever $\geq 37 / 8^{\circ} \mathrm{C}$, cough, with clinical or radiological evidence of pneumonia or acute respiratory distress) and preliminarily diagnosed as COVID-19 were isolated as soon as possible. Patients who did not require hospitalization, voluntary home isolation was recommended. This strategy provided more resources in our centre to handle only those cases which required hospital health professional care especially the most critically affected patients. In some developed countries, using telemedicine was very common before the advent of COVID-19. Telemedicine triage at emergency rooms plays a significant role in reducing the risk of contamination of health professionals and provide better precaution for critically ill patients. ${ }^{4}$

\section{Operating room (OR) management}

In our centre, we have four ORs, including general (multidisciplinary surgery theatre), orthopaedic surgery, cardiovascular open-heart surgery, as well as burns plastic and reconstructive surgery. For decreasing the risk of viral transmission and polluting other ORs, we just used general OR. Moreover, we decided to postpone all the elective procedures for two reasons: first, to focus on pandemic circumstances and not to waste our resources and energy. Second, since asymptomatic individuals can transmit the virus, there were ongoing efforts to identify and manage infected patients to decrease the risk of infection transmission in OR and other sections of our centre were non-elective, but emergent non-COVID-19 patients were admitted. In this regards, Singapore has made the same approach for increasing capacity of their hospital wards and cancel all the elective surgeries. ${ }^{5}$ For emergency surgeries, we took some steps to reduce the risk of perioperative COVID-19 transmission for both non-COVID-19 patients and health care staff. For example, applying a surgical mask for all patients who entered the OR, single-use anaesthesia devices were preferred where possible, after surgery all the devices (including anaesthetic machines, stethoscope, etc.) and surfaces were disinfectant by $70 \%$ ethanol and sterile processing was applied for instruments decontamination under strict protective conditions. Besides, after all, procedures an extra time was dedicated to disinfecting the surgery rooms and using purifying air conditioning. In comparison with our protocols, many institutions have used other methods for disinfection process such as using $62-71 \%$ ethanol, $0.5 \%$ hydrogen peroxide or $0.1 \%$ sodium hypochlorite, sodium hypochlorite followed by hydrogen peroxide vaporization, ammonium chloride, as well as ultraviolet-C irradiation which are all confirmed by WHO. ${ }^{5,6}$ For many infectious surgeries such as COVID-19, negative pressure ventilation rooms is highly recommended. ${ }^{5,7}$ However, in our centre, we have positive pressure ventilation and we should consider this kind of ventilation soon.

\section{Anaesthesia management}

Anesthesiologists and anaesthesia staff members are at the highest risk of COVID-19 infection because one way to transmit this virus is by respiratory droplets and contact routes. As a result, tracheal intubation is the highest risk of transmission. If it is possible, regional anaesthesia is preferred to general anaesthesia. ${ }^{5}$ For other patients who need general anaesthesia, it is very crucial to consider some cautions such as using an antiviral filter to connect between patients and anaesthetic machine (to reduce the risk of contamination), a well-fitted face mask (for pre-oxygenation) and intubation with sufficient relaxation as well as doing video laryngoscopy by an expert anesthesiologist are highly recommended to decrease the risk of interaction with patient's airway during intubation. In patients with confirmed COVID-19, awake intubation has been avoided in many literatures ${ }^{4,5,8}$ to reduce the risk of extra contaminating anesthesiologists and medical staff, which are following our strategies.

\section{Admission to ICU}

Patients who do not respond appropriately to standard oxygen therapies mostly require advanced oxygen therapy or ventilator support. We provide a separate space (a distance of at least $1 \mathrm{~m}$ between patients) with adequate environmental ventilation for patients who need special support. The protocol for cleaning and disinfection of contaminated surfaces as well as using video-laryngoscope are the same as OR. While in ICU, we have used video laryngoscopy for intubation of patients which has used generally in many hospitals, some other protective device like neonatal incubator hood have been invented for reducing the risk of contamination during airway management. ${ }^{9}$

\section{Personnel protective equipment (PPE)}

During the pandemic, it is vital to protect health care staff who are interacting with patients. Standards need to be taken for all cases of known or suspected COVID-19. Our strategies in this regard were provided personal protective equipment (PPE) including gloves, gowns, face shield, surgical mask (for routine care) and N95 mask (for confirmed and suspected cases) which were indispensable to protect health care staff from infectious disease spread. In many countries, in addition to PPE, a powered air-purifying respirator (PAPAR) is used to protect health care staffs and doctors who were involved in airway management. Although it has been reported that, PAPAR guarantee superior protection, in our centre it was not possible to provide PAPAR and we have used surgical mask or N95 instead.

\section{CONCLUSION}

According to provincial statistics until September 15, 2020, we had 739 cases died from this infectious disease. Until now, we had some cases among health care workers (who are infected with COVID-19) and unfortunately, three nurses died during this pandemic. Although all these three nurses worked in dedicated wards for caring COVID-19 patients, it was challenging to conclude whether nurses became sick at work or in another place.

In comparison with our study, one survey has done in February in China, indicated that among 1,716 confirmed tests, five deaths occurred among health care workers. ${ }^{10}$ However, by using appropriate PPE, the rate of nurses infected by COVID-19 declined dramatically at the end of February. In another study, which had 106 deaths among staffs, none of them was anesthesiologists or intensive care doctors. ${ }^{11}$ Of note, airway management, as well as intubation, are vital in the management of COVID-19 critically ill patients and as a result, anaesthetists, play a crucial role in this section. Fortunately, in our department, we do not have any report about mortality among our anaesthetists and medical staffs yet. We hope that by following the standards and using appropriate PPE, we can protect our health care workers more and overcome COVID-19 pandemic.

Albeit we still have a lot to learn, all health care systems must be aware of the potential impact of any infectious disease and follow the guidelines to protect their medical staffs and act appropriately to overcome any pandemic.

\section{SUMMARY}

In summary, we need to learn about any previous infectious disease and prepare all hospitals as well as infrastructures to manage all kind of 
infectious outbreak. Moreover, health care systems should support and protect all medical staffs to cope with any infectious disease.

\section{ACKNOWLEDGEMENT}

The authors thank all the members of anaesthesia department, anaesthetic technicians, nurses, surgeons and medical doctors in Payambar Azam medical centre for their suggestions and excellent role in caring patients.

\section{CONFLICT OF INTEREST}

The authors declare that there are no conflicts of interest.

\section{ABBREVIATIONS}

COVID-19: Coronavirus disease 2019; ICU: Intensive care unit; OR: Operating room; PPE: Personnel protective equipment; WHO: World health organization.

\section{REFERENCES}

1. Lie SA, Wong SW, Wong LT, Wong TGL, Chong SY. Practical considerations for performing regional anesthesia: lessons learned from the COVID-19 pandemic. Canadian Journal of Anesthesia. 2020;67:885-92

2. Nicola M, O'Neill N, Sohrabi C, Khan M, Agha M, Agha R. Evidence based management guideline for the COVID-19 pandemic-review article. International
Journal of Surgery. 2020;77:206-16

3. Coronavirus Disease (COVID-19) Situation Reports. 2020. https://www.who.int/ emergencies/diseases/novel-coronavirus-2019/situation-reports

4. Quintão VC, Simões CM, ELima LHN, DeBarros GAM, Salgado-Filho MF, Guimarães GMN, et al. The anesthesiologist and COVID-19. Brazilian Journal of Anesthesiology. 2020;70(2):77-81.

5. Wong J, Goh QY, Tan Z, Lie SA, Tay YC, Ng SY, et al. Preparing for a COVID-19 pandemic: A review of operating room outbreak response measures in a large tertiary hospital in Singapore. Canadian Journal of Anesthesia. 2020;67:732-45.

6. Song X, Vossebein L, Zille A. Efficacy of disinfectant-impregnated wipes used for surface disinfection in hospitals: A review. Antimicrobial Resistance and Infection Control. 2019;8(1):139.

7. Brewster DJ, Chrimes NC, Do TB, Fraser K, Groombridge CJ, Higgs A, et al. Consensus statement: Safe Airway Society principles of airway management and tracheal intubation specific to the COVID-19 adult patient group. Med J Aust. 2020;16.

8. Coronavirus disease 2019 (COVID-19): update for anesthesia. 2020. https:// scholar.google.com/scholar?hl=en\&as_sdt=0\%2C5\&q=Coronavirus+disease+ 2019+\%28COVID-19\%29\%3A+update+for+anesthesiologists+and+intensivis ts+March $+2020 \& b \operatorname{tnG}=$

9. Rahmoune FC, Yahia MMB, Hajjej R, Pic S, Chatti K. Protective Device during Airway Management in Patients with Coronavirus Disease 2019 (COVID-19). Anesthesiology: The Journal of the American Society of Anesthesiologists. 2020(133):473-5.

10. Novel CPERE. The epidemiological characteristics of an outbreak of 2019 nove coronavirus diseases (COVID-19) in China. Zhonghua liu xing bing xue za zhi= Zhonghua liuxingbingxue zazhi. 2020;41(2):145-51.

11. Kursumovic E, Lennane S, Cook TM. Deaths in healthcare workers due to COVID-19: the need for robust data and analysis. Anaesthesia. 2020;75(8):989-92.

Cite this article : Jarineshin H, Fekrat F, Dalfardi B, Khosravi F. A Brief Report about Managing the Spread of COVID-19 with Limited Resources in Bandar Abbas City, South of Iran. BEMS Reports. 2020;6(2):28-30. 